\title{
GENERIC FRÉCHET-DIFFERENTIABILITY AND PERTURBED OPTIMIZATION PROBLEMS IN BANACH SPACES $\left({ }^{1}\right)$
}

\author{
BY \\ IVAR EKELAND AND GÉRARD LEBOURG
}

\begin{abstract}
We define a function $F$ on a Banach space $V$ to be locally e-supported by $u^{*} \in V^{*}$ at $u \in V$ if there exists an $\eta>0$ such that $\|v-u\|<$ $\eta \Rightarrow F(v) \geqslant F(u)+\left\langle u^{*}, v-u\right\rangle-\epsilon\|v-u\|$. We prove that if the Banach space $V$ admits a nonnegative Fréchet-differentiable function with bounded nonempty support, then, for any $\epsilon>0$ and every lower semicontinuous function $F$, there is a dense set of points $u \in V$ at which $F$ is locally $\epsilon$-supported. The applications are twofold. First, to the study of functions defined as pointwise infima; we prove for instance that every concave continuous function defined on a Banach space with Fréchet-differentiable norm is Fréchet-differentiable generically (i.e. on a countable intersection of open dense subsets). Then, to the study of optimization problems depending on a parameter $u \in V$; we give general conditions, mainly in the framework of uniformly convex Banach spaces with uniformly convex dual, under which such problems generically have a single optimal solution, depending continuously on the parameter and satisfying a first-order necessary condition.
\end{abstract}

1. Local $\epsilon$-supports. Let $V$ be a Banach space and $V^{*}$ its topological dual. The canonical bilinear form on $V \times V^{*}$ will be denoted by brackets $\langle\cdot, \cdot\rangle$, the norm of $V$ by $\|\cdot\|$, the norm of $V^{*}$ by $\|\cdot\|_{*}$. Let $F: V \rightarrow R \cup\{+\infty\}$ be a function on $V$; recall that the effective domain of $F$ is denoted by dom $F$ and is defined as the set of points where $F$ is finite:

$$
\operatorname{dom} F=\{v \mid F(v)<+\infty\}
$$

Definition 1.1. A continuous linear functional $u^{*} \in V^{*}$ is locally $\epsilon$-supporting to $F$ at $u$ iff $F(u)<+\infty$ and there exists an $\eta>0$ such that

$$
\|v-u\| \leqslant \eta \Rightarrow F(v) \geqslant F(u)+\left\langle u^{*}, v-u\right\rangle-\epsilon\left\|_{v}-u\right\| .
$$

The set of continuous linear functionals, $\epsilon$-supporting to $F$ at $u$, will be called the e-support of $F$ at $u$, and denoted by $S_{\epsilon} F(u)$. If it is nonempty, we shall say that $F$ is locally e-supported at $u$.

The following are easy consequences of the definition:

Received by the editors August 7, 1975.

AMS (MOS) subject classifications (1970). Primary 46B99, 49A25; Secondary 26A54.

$\left({ }^{1}\right)$ The main results of this paper have been announced as a Compte Rendu de l'Académie des Sciences de Paris (June 23, 1975). 


$$
S_{\epsilon} F(u)=\varnothing \text { if } u \notin \operatorname{dom} F .
$$

$$
\begin{gathered}
S_{\epsilon} F(u) \text { is a convex subset of } V^{*} . \\
S_{\epsilon} F(u)+S_{\theta} G(u) \subset S_{\epsilon+\theta}(F+G)(u) . \\
S_{\epsilon} F(u) \subset S_{\theta} F(u) \forall \theta \geqslant \epsilon . \\
u^{*} \in \bigcap_{\epsilon>0} S_{\epsilon} F(u) \cap-S_{\epsilon}(-F)(u) \Leftrightarrow u^{*}=F^{\prime}(u) \text { (Fréchet-derivative). } \\
S_{\epsilon} F(u) \neq \varnothing \Rightarrow F \text { is lower semicontinuous at } u .
\end{gathered}
$$

This last relation is proved by noting that in (1.2) the right-hand side is a lower semicontinuous function of $v$ which coincides with $F$ at $u$.

Let us now state the main result. We shall need an assumption on the Banach space $V$ :

$$
\text { There exists on } V \text { a nonnegative continuous function } \Psi
$$
which is zero outside some bounded set, and Fréchetdifferentiable at every point where it is nonzero.

This means that $\Psi$ is $\geqslant 0$ but is zero outside some ball $B$ centered at the origin. By translation, we can assume that $\Psi(0)>0$. By homothety, we can assume the ball $B$ to be as small as need be. In the sequel, we shall use the function $\Phi=1 / \Psi$. It is well defined and lower semicontinuous as a mapping from $V$ to $R \cup\{+\infty\}$, and it is Fréchet-differentiable on its effective domain.

THEOREM 1.2. Let $\epsilon>0$ be given. If the Banach space $V$ satisfies condition $(\mathrm{H})$, then every lower semicontinuous function $F$ is locally $\epsilon$-supported at all points of a dense subset of $\operatorname{dom} F$.

Proof. Let there be given a point $u_{0} \in \operatorname{dom} F$, a neighbourhood $W$ of the origin in $V$, and let us find in $u_{0}+W$ a point where $F$ is locally $\epsilon$-supported.

As $F$ is lower semicontinuous, we can find a smaller neighbourhood of the origin $V \subset W$ such that $F$ is bounded from below on $u_{0}+V$ :

$$
\exists m: \forall u \in u_{0}+V, \quad F(u) \geqslant m .
$$

Take a Fréchet-differentiable function $\Psi \geqslant 0$ with support contained in $V$, and define functions $\Phi$ and $G$ on $V$ by

$$
\begin{aligned}
& \Phi(u)=\Psi\left(u-u_{0}\right)^{-1}, \\
& G(u)=F(u)+\Phi(u) .
\end{aligned}
$$

The function $G$ is lower semicontinuous and bounded from below on the Banach space $V$. By Theorem 1.1 of [11], with $\lambda=2$, there exists a point $u_{\epsilon}$ such that 


$$
\forall u \in V, \quad G(u) \geqslant G\left(u_{\epsilon}\right)-1 / 2 \epsilon\left\|u_{\epsilon}-u\right\| .
$$

This implies that $0 \in S_{\epsilon / 2} G\left(u_{\epsilon}\right)$. Let $u^{*}$ be the Fréchet-derivative of $-\Phi$ at $u_{\epsilon}$. By (1.7) we know that $u^{*} \in S_{\epsilon / 2}-\Phi\left(u_{\epsilon}\right)$. This implies by (1.5) that

$$
u^{*} \in S_{\epsilon}(G-\Phi)\left(u_{\epsilon}\right)=S_{\epsilon} F\left(u_{\epsilon}\right) .
$$

Moreover, we conclude from (1.11) that

$$
\operatorname{dom} G=\operatorname{dom} F \cap \operatorname{dom} \Phi \subset \operatorname{dom} F \cap\left(u_{0}+V\right) .
$$

Since $u_{\epsilon} \in \operatorname{dom} G$, our claim is proved.

Condition $(\mathrm{H})$ is satisfied whenever the Banach space $V$ admits an equivalent norm which is Fréchet-differentiable on $V \backslash\{0\}$; to see this, just take a nonnegative function $\varphi$ on $R \backslash\{0\}$ with compact nonempty support, and define $\Phi(u)$ $=\varphi(\|u\|)$. A converse has been proved for weakly compactly generated spaces [13].

Typically, Hilbert spaces, $L^{p}$ spaces $(1<p<\infty)$, Banach spaces with separable dual, all satisfy condition $(\mathrm{H}) ; l^{1}$ and $l^{\infty}$ do not, nor does any space which contains one of them isomorphically.

THEOREM 1.3. The following properties are equivalent:

(1.15) the function $F$ is Fréchet-differentiable at $u \in V$,

(1.16) for every $\epsilon>0$, both $F$ and $-F$ are $\epsilon$-supported at $u$.

Proof. It is clear from (1.7) that (1.15) implies (1.16). Let us prove the converse. Assuming (1.16), we replace $\epsilon$ by $1 / n$ and $F$ by $-F$ in formula (1.2) to get

$$
\begin{aligned}
& \forall n \in N, \exists \eta_{n}>0, \exists u_{n}^{*} \in V^{*}: \\
& \|v-u\| \leqslant \eta_{n} \Rightarrow F(v) \geqslant F(u)+\left\langle u_{n}^{*}, v-u\right\rangle-\left\|_{v}-u\right\| / n, \\
& \forall n \in N, \quad \exists \epsilon_{n}>0, \exists v_{n}^{*} \in V^{*}: \\
& \left\|_{v}-u\right\| \leqslant \epsilon_{n} \Rightarrow F(v) \leqslant F(u)+\left\langle v_{n}^{*}, v-u\right\rangle+\left\|_{v}-u\right\| / n
\end{aligned}
$$

Take any $m \geqslant n$, write (1.17) for $m$, and compare it with (1.18). This yields

$$
\left\|_{v}-u\right\| \leqslant \min \left(\eta_{m}, \epsilon_{n}\right) \Rightarrow\left\langle u_{m}^{*}-v_{n}^{*}, v-u\right\rangle \leqslant 2\left\|_{v}-u\right\| / n
$$

and hence

$$
\forall n \in N, \quad \forall m \geqslant n, \quad\left\|u_{m}^{*}-v_{n}^{*}\right\|_{*} \leqslant 2 / n .
$$

Similarly, writing (1.18) for $m$ and comparing it to (1.17) 


$$
\forall n \in N, \quad \forall m \geqslant n, \quad\left\|u_{n}^{*}-v_{m}^{*}\right\|_{*} \leqslant 2 / n .
$$

This proves that both $u_{n}$ and $v_{n}$ are Cauchy sequences converging (as $V^{*}$ is complete) to the same limit $u^{*} \in V^{*}$. We claim that $u^{*}$ is the Fréchet-derivative of $F$ at $u$.

To see this, take any $\epsilon>0$. Choose $n \geqslant 3 / \epsilon$. Let $\eta$ be $\min \left(\eta_{n}, \epsilon_{n}\right)$. By (1.17) and (1.18), $\|v-u\| \leqslant \eta$ implies that

$$
\left\langle u_{n}^{*}, v-u\right\rangle-\|v-u\| / n \leqslant F(v)-F(u) \leqslant\left\langle v_{n}^{*}, v-u\right\rangle+\|v-u\| / n .
$$

Letting $m \rightarrow \infty$ in (1.20) and (1.21), we get

$$
\left\|u_{n}^{*}-u^{*}\right\|_{*} \leqslant 2 / n \text { and }\left\|v_{n}^{*}-u^{*}\right\|_{*} \leqslant 2 / n \text {. }
$$

Replacing $u_{n}^{*}$ and $v_{n}^{*}$ by $u^{*}$ in (1.22), we get (1.24) $\left\langle u^{*}, v-u\right\rangle-3\|v-u\| / n \leqslant F(v)-F(u) \leqslant\left\langle u^{*}, v-u\right\rangle+3\|v-u\| / n$.

As $3 / n \leqslant \epsilon$, this is exactly the Fréchet-differentiability of $F$ at $u$, and concludes our proof.

For the next corollary, recall that a $G_{\delta}$ is a countable intersection of open subsets.

COROLLARY 1.4. Assume that, for every $\epsilon>0$, the set of points where $F$ is locally $\epsilon$-supported is a $G_{\delta}$; likewise for $-F$. Assume also that $F$ is continuous on $V$. Then $F$ is Fréchet-differentiable at every point of a dense $G_{\delta}$ subset of $V$.

Proof. As $F$ is continuous, both $F$ and $-F$ are lower semicontinuous. By Theorem 1.2, the set of points where $F$ is locally $1 / n$-supported is dense in $V$; let us denote it by $T_{n}^{+}$. By assumption, it is a $G_{\delta}$. Likewise, denote by $T_{n}$ the set of points where $-F$ is locally $1 / n$-supported: it is a dense $G_{\delta}$.

Let $T=\bigcap_{n=1}^{\infty}\left(T_{n}^{+} \cap T_{n}^{-}\right)$. By the Baire category theorem, $T$ is again a dense $G_{\delta}$. But $T$ is just the set of points where $F$ and $-F$ are locally $\epsilon$-supported for every $\epsilon>0$, and the result follows by (1.7).

The following remarks are due to Frank H. Clarke (private communication):

REMARK 1.5. There is another (equivalent) way of stating Definition 1.1: $u \in V$ is locally $\epsilon$-supporting at $u \in V$ iff the function

$$
v \mapsto F(v)-\left\langle u^{*}, v\right\rangle+\epsilon\|v-u\|
$$

attains at $u$ a local minimum.

REMARK 1.6. Let $V$ be any Banach space, and $F$ a lower semicontinuous function on $V$. Then there is a dense set of points $u \in V$ with the following "vertical cone" property:

$$
\exists \eta>0, \quad \exists k>0:\|v-u\| \leqslant \eta \Rightarrow F(v) \geqslant F(u)-k\left\|_{v}-u\right\| .
$$


Clearly (1.2) implies (1.26), but (1.26) holds even when the Banach space does not satisfy condition (H). The proof is the same as for Theorem 1.2, using for $\Phi$ the function

$$
\begin{aligned}
& \Phi(u)=\left(\eta-\left\|u-u_{0}\right\|\right)^{-1} \text { if }\left\|u-u_{0}\right\|<\eta, \\
& \Phi(u)=+\infty \text { otherwise, }
\end{aligned}
$$

where $\eta>0$ is chosen so small that the ball of radius $\eta$ lies inside the prescribed neighbourhood $V$. The function $-\Phi$ is no longer differentiable, but it is easily seen to have the vertical cone property at every point of its effective domain. Relation (1.12) can be written:

$$
\forall u \in \operatorname{dom} \Phi, \quad F(u) \geqslant F\left(u_{\epsilon}\right)+\Phi\left(u_{\epsilon}\right)-\Phi(u)-\frac{\epsilon}{2}\left\|u-u_{\epsilon}\right\| .
$$

The right-hand side is a function which has the vertical cone property and coincides with $F$ at $u_{\epsilon}$. Hence (1.28) implies that $F$ itself has the vertical cone property at $u_{\epsilon}$.

2. Application to functions defined as pointwise infima. Introduce now an abstract set $X$, and a function

$$
f: V \times X \rightarrow \mathbf{R} .
$$

We define a function $F$ on $V$ by

$$
F(u)=\inf _{x \in X} f(u, x)
$$

assuming always that this infimum is well defined:

$$
F(u)>-\infty \quad \forall u \in V .
$$

Definition 2.1. Fix $u \in V$. Associate with every $\theta>0$ the subset

$$
A_{\theta}=\{x \in X \mid f(u, x) \leqslant F(u)+\theta\} .
$$

The family $A_{\theta}, \theta>0$, of subsets form the basis of a filter $F_{u}$ on $X$, called the minimizing filter of $f$ at $u$.

We refer the reader to [6] for information about filters. In the present case, it is sufficient to check that $F_{u}$ is nonempty and does not contain the empty subset, and that the intersection of two members of $F_{u}$ contains a member of $F_{u}$. Both properties follow at once from the definition; in particular, it follows from (2.4) that

$$
A_{\epsilon} \cap A_{\theta}=A_{\min (\epsilon, \theta)} \text {. }
$$

We now apply Definition 1.1 to this situation.

Proposition 2.2. Assume that 


\section{$F$ is locally $\epsilon$-supported at $u$}

and that there exist a subset $A \in F_{u}$ of $X$ and a neighbourhood $U$ of $u$ in $V$ such that

$$
\begin{gathered}
\forall x \in A, \quad v \mapsto f(v, x) \text { is Fréchet-derivable at } u . \\
\left\{v \mapsto f_{v}^{\prime}(v, x) \mid x \in A\right\} \text { is equicontinuous at } u .
\end{gathered}
$$

Then there exists a subset $C \in F_{u}$ of $X$ and a neighbourhood $V$ of $u$ in $V$ such that

$$
\text { diameter }\left\{f_{v}^{\prime}(v, x) \mid v \in V, x \in C\right\} \leqslant 8 \epsilon \text { in } V^{*} \text {-norm. }
$$

Proof. Let $u^{*} \in S_{\epsilon} F(u)$. There exists an $\eta_{1}>0$ such that

$$
\|u-v\| \leqslant \eta_{1} \Rightarrow F(v) \geqslant F(u)+\left\langle u^{*}, v-u\right\rangle-\epsilon\|u-v\| .
$$

By (2.8) we can find an $\eta_{2}<\eta_{1}$ such that

$$
\|u-v\| \leqslant \eta_{2} \Rightarrow\left\|f_{v}^{\prime}(u, x)-f_{v}^{\prime}(v, x)\right\|_{*} \leqslant \epsilon \quad \forall x \in A .
$$

Note that

$$
f(v, x)=\int_{0}^{1}\left\langle f_{v}^{\prime}(u+t(v-u), x), v-u\right\rangle d t+f(u, x)
$$

so that (2.12) implies

(2.14) $\|u-v\| \leqslant \eta_{2} \Rightarrow f(v, x) \leqslant f(u, x)+\left\langle f_{v}^{\prime}(u, x), v-u\right\rangle+\epsilon\|v-u\| \quad \forall x \in A$.

Remember now that $A$ belongs to the minimizing filter $F_{u}$ at $u$, and this means that it contains a subset $A_{\theta}$ as in (2.4). Using (2.5), we may assume $\theta \leqslant \epsilon \eta_{2}$, so that

$$
\forall x \in A_{\theta}, \quad f(u, x) \leqslant F(u)+\epsilon \eta_{2} .
$$

Remember also that, by (2.2)

$$
F(v) \leqslant f(v, x)
$$

Adding (2.14), (2.15) and (2.16) together, we obtain

$$
\|u-v\| \leqslant \eta_{2} \Rightarrow F(v) \leqslant F(u)+\left\langle f_{v}^{\prime}(u, x), v-u\right\rangle+2 \epsilon \eta_{2} .
$$

Adding the inequalities (2.11) and (2.17), we obtain

$$
\begin{gathered}
\|u-v\| \leqslant \eta_{2} \Rightarrow\left\langle u^{*}-f_{v}^{\prime}(u, x), v-u\right\rangle \leqslant 3 e \eta_{2}, \\
\sup _{w w \| \eta_{2}}\left\langle u^{*}-f_{v}^{\prime}(u, x), w\right\rangle \leqslant 3 e \eta_{2},
\end{gathered}
$$




$$
\left\|u^{*}-f_{v}^{\prime}(u, x)\right\|_{*} \leqslant 3 \epsilon .
$$

By the triangle inequality, we obtain from (2.12) and (2.20)

$$
\|u-v\| \leqslant \eta_{2} \Rightarrow\left\|u^{*}-f_{v}^{\prime}(v, x)\right\|_{*} \leqslant 4 \epsilon .
$$

Thus the set $\left\{f_{v}^{\prime}(v, x) \mid\|v-u\| \leqslant \eta_{2}, x \in A_{\theta}\right\}$ is contained in a ball of radius $4 \epsilon$ centered at $u^{*}$. This implies that its diameter is less than $8 \epsilon$ :

$$
\begin{aligned}
& \|u-v\| \leqslant \eta_{2}, \quad x \in A_{\theta} \\
& \|u-w\| \leqslant \eta_{2}, \quad y \in A_{\theta}
\end{aligned} \Rightarrow\left\|f_{v}^{\prime}(v, x)-f_{v}^{\prime}(w, y)\right\|_{*} \leqslant 8 \epsilon .
$$

Note that the family of subsets of $V^{*}$

$$
A_{u}^{*}(\eta, \theta)=\left\{f_{v}^{\prime}(v, x) \mid\left\|_{v}-u\right\| \leqslant \eta, f(u, x) \leqslant F(u)+\theta\right\}, \quad \eta>0, \theta>0
$$

is a filter basis. Let us associate with the point $u \in V$ the filter $G_{u}$ on $V^{*}$ generated by the $A_{u}^{*}(\eta, \theta)$.

Denote by $T_{\epsilon}$ the set of points $u \in V$ such that the filter $G_{u}$ has an element of diameter less than $\epsilon$.

$$
u \in T_{\epsilon} \Leftrightarrow \exists \eta>0, \exists \theta>0: \text { diameter } A_{u}^{*}(\eta, \theta) \leqslant \epsilon .
$$

Proposition 2.3. Assume that

$$
u \in T_{\epsilon}
$$

and that there exists $\alpha>0$ and $\beta>0$ such that

$$
\left\{f_{v}^{\prime}(v, x) \mid\left\|_{v}-u\right\| \leqslant \alpha, f(v, x) \leqslant F(u)+\beta\right\} \text { is bounded in } V^{*} .
$$

Then $T_{\epsilon}$ is a neighbourhood of $u$.

Proof. By (2.25) there exist $\eta>0$ and $\theta>0$ such that the diameter of $A_{u}^{*}(\eta, \theta)$ is less than $\epsilon$. The proof goes by showing that for all $v$ in a certain neighbourhood of $u$, the set $A_{u}^{*}(\eta, \theta)$ contains an element of $G_{v}$.

Denote $\alpha^{\prime}=\min (\alpha, \eta)$ and $\beta^{\prime}=\min (\beta, \theta)$.

Note first that the $f(\cdot, x)$ are continuous, and that $F$ is their pointwise infimum. Hence $F$ must be upper semicontinuous. We can find $\gamma>0$ so small that

$$
\|v-u\| \leqslant \gamma \Rightarrow F(v) \leqslant F(u)+\beta^{\prime} / 3 .
$$

Note also that, by (2.26), there is a constant $c$ such that

$$
\|v-u\| \leqslant \alpha^{\prime} \text { and } f(v, x) \leqslant F(u)+\beta^{\prime} \Rightarrow\left\|f_{v}^{\prime}(v, x)\right\|_{*} \leqslant c .
$$

Take now a positive number $\delta \leqslant \min \left(\gamma, \alpha^{\prime} / 2, \beta^{\prime} / 3 c\right)$. We claim that the 
ball or radius $\delta$ centered at $u$ lies entirely inside $T_{\epsilon}$, i.e. $\left\|_{v}-u\right\| \leqslant \delta \Rightarrow v \in T_{\epsilon}$. Indeed, let $w \in V$ and $x \in X$ be such that

$$
\|w-v\| \leqslant \delta \text { and } f(v, x) \leqslant F(v)+\beta^{\prime} / 3 .
$$

This implies that $\|w-u\| \leqslant \alpha^{\prime}$ and, by adding (2.27)

$$
f(v, x) \leqslant F(u)+2 \beta^{\prime} / 3 \text {. }
$$

Now consider the real function $\varphi(t)=f(v+t(v-w), x)-f(v, x)$ with derivative

$$
\varphi^{\prime}(t)=\left\langle f_{v}^{\prime}(v+t(v-w)), v-w\right\rangle .
$$

Now, $\varphi(0)=0$. Let $t_{0} \in[0,1]$ be the first instant where $\varphi(t) \geqslant \beta^{\prime} / 3$. Using (2.28)

$$
\begin{aligned}
\varphi\left(t_{0}\right) & =\int_{0}^{t_{0}} \varphi^{\prime}(t) d t \leqslant c t_{0}\|v-w\|, \\
\beta^{\prime} / 3 & \leqslant c t_{0} \delta .
\end{aligned}
$$

Hence $t_{0} \geqslant 1$. Using the fact that $\varphi(1)=f(w, x)-f(v, x)$, this yields

$$
f(w, x) \leqslant f(v, x)+c\|v-w\| .
$$

Adding (2.30) and (2.34), and noting that $\|w-v\| \leqslant \delta \leqslant \beta^{\prime} / 3 c$ :

$$
f(w, x) \leqslant F(u)+\beta^{\prime}
$$

which is true for any $w$ such that $\|w-v\| \leqslant \delta$. Particularizing $w$ at $u$, we finally get

$$
\left\|_{v}-u\right\| \leqslant \delta \Rightarrow A_{v}^{*}\left(\delta, \beta^{\prime} / 3\right) \subset A_{u}^{*}\left(\alpha^{\prime}, \beta^{\prime}\right) \subset A_{u}^{*}(\eta, \theta) .
$$

If the right-hand side of the inclusion has diameter less than $\epsilon$, then so does the left-hand side. This concludes the proof.

We thus have some information on the behavior of the filter $G_{u}$. We shall translate it in terms of the behaviour of $F$ at $u$ by use of the following

Proposition 2.4. If assumptions (2.7), (2.8) and (2.26) are satisfied, then $F$ is lipschitzian on some neighbourhood of $u$. If moreover the filter $G_{u}$ converges to $u^{*}$ in $V^{*}$-norm, then $F$ admits $u^{*}$ as Fréchet-derivative at $u$.

Proof. The first assertion is a consequence of (2.26). Indeed, by the preceding proof, there is a $\delta>0$ such that (2.34) holds

$$
\|u-v\| \leqslant \delta \text { and }\|v-w\| \leqslant \delta \Rightarrow f(w, x) \leqslant f(v, x)+c\|w-v\| \text {. }
$$

Exchanging the roles of $v$ and $w$, we get likewise 
(2.38) $\|u-w\| \leqslant \delta$ and $\|w-v\| \leqslant \delta \Rightarrow f(v, x) \leqslant f(w, x)+c\|v-w\|$.

Hence, by adding the inequalities:

(2.39) $\|u-v\| \leqslant \frac{\delta}{2}$ and $\|u-w\| \leqslant \frac{\delta}{2} \Rightarrow|f(w, x)-f(v, x)| \leqslant c\|v-w\|$.

Now for the second assertion. Let $\epsilon>0$ be given.

Let $x_{n}$ be a minimizing sequence in $X$ at $u$ :

$$
f\left(u, x_{n}\right) \rightarrow F(u) .
$$

By (2.8) there is a $p \in \mathrm{N}$ and an $\eta>0$ such that $n \geqslant p$ and $\|v-u\| \leqslant \eta$ imply (compare (2.14))

$$
f\left(u, x_{n}\right)+\left\langle f_{v}^{\prime}\left(u, x_{n}\right), v-u\right\rangle+\epsilon\left\|_{v}-u\right\| \geqslant f\left(v, x_{n}\right) .
$$

By assumption, the sequence $f_{v}^{\prime}\left(u, x_{n}\right)$ converges to $u^{*}$ in $V^{*}$. Choose a $q \geqslant p$ sufficiently large for $\left\|u^{*}-f_{v}^{\prime}\left(u, x_{n}\right)\right\|_{*} \leqslant \epsilon$ for every $n \geqslant q$, and write it into (2.41):

$$
f\left(u, x_{n}\right)+\left\langle u^{*}, v-u\right\rangle+2 \epsilon\|v-u\| \geqslant f\left(v, x_{n}\right)
$$

for $\|v-u\| \leqslant \eta$ and $n \geqslant q$. Letting $n$ go to infinity, and taking into account (2.40) and the inequality $f(v, x) \geqslant F(v)$, we get the first half of the Fréchetdifferentiability formula:

$$
\|v-u\| \leqslant \eta \Rightarrow F(u)+\left\langle u^{*}, v-u\right\rangle+2 \epsilon\|v-u\| \geqslant F(v) .
$$

To get the other half, take $\eta>0$ and $\theta>0$ such that

$$
\begin{aligned}
& \|u-v\| \leqslant \eta \text { and } f(u, x) \leqslant F(u)+\theta \Rightarrow\left\|f_{v}^{\prime}(u, x)-u^{*}\right\|_{*} \leqslant \epsilon \\
& \text { and } f(v, x) \geqslant f(u, x)+\left\langle f_{v}^{\prime}(u, x), v-u\right\rangle-\epsilon\|v-u\| .
\end{aligned}
$$

We have just seen $((2.29) \Rightarrow(2.35)$ with $w=u)$ that there exists a $\delta>0$ such that $\|u-v\| \leqslant \delta$ and $f(v, x) \leqslant F(v)+\theta / 3$ imply $\|u-v\| \leqslant \eta$ and $f(u, x) \leqslant F(u)+\theta$. Writing that into $(2.44)$ we get

$$
\begin{aligned}
\|u-v\| \leqslant \delta \text { and } f(v, x) \leqslant F(v)+\theta / 3 \\
\quad \Rightarrow f(v, x) \geqslant f(u, x)+\left\langle u^{*}, v-u\right\rangle-2 \epsilon\|v-u\| .
\end{aligned}
$$

This formula holds for all points $x_{n}$ of a minimizing sequence at $v$ (at least for $n$ sufficiently large). Letting $n$ go to infinity, $f\left(v, x_{n}\right) \rightarrow F(v)$, and we obtain

$$
\|u-v\| \leqslant \delta \Rightarrow F(v) \geqslant F(u)+\left\langle u^{*}, v-u\right\rangle-2 \epsilon\left\|_{v}-u\right\| .
$$

Together with (2.43), this completes the proof. $\square$

We now state our main result. Let us first, for the reader's convenience, 
recall the definitions of the filters involved. With every point $u \in V$ we associate the filters $F_{u}, G_{u}, H_{u}$, on $V, V^{*}, V^{*}$, respectively generated by the subsets:

$$
\begin{aligned}
A_{\theta} & =\{x \in X \mid f(u, x) \leqslant F(u)+\theta\}, \\
A_{\theta, \eta}^{*} & =\left\{f_{v}^{\prime}(v, x) \mid\left\|_{v}-u\right\| \leqslant \eta, f(u, x) \leqslant F(u)+\theta\right\}, \\
B_{\theta, \eta}^{*} & =\left\{f_{v}^{\prime}(v, x) \mid\left\|_{v}-u\right\| \leqslant \eta, f(v, x) \leqslant F(u)+\theta\right\},
\end{aligned}
$$

with the convention that if $f(\cdot, x)$ is not Fréchet-differentiable at $u$, then $f_{v}^{\prime}(u, x)$ is considered to be the whole space $V^{*}$.

THEOREM 2.5. Let $V$ be a Banach space satisfying condition $(\mathrm{H}), X$ an abstract set, and $f$ a function on $V \times X$ such that

$$
\forall u \in V, \quad F(u)=\inf _{x \in X} f(u, x)>-\infty .
$$

Assume there is an open subset $\Omega \subset V$ every point $u$ of which has the following property:

$$
\begin{aligned}
& \text { there is a } B_{\eta, \theta}^{*} \in H_{u} \text { which is norm-bounded in } V^{*} \text {, } \\
& \text { there is an } A_{\theta} \in F_{u} \text { such that }\left\{f_{v}^{\prime}(\cdot, x) \mid x \in A\right\} \text { is an } \\
& \text { equicontinuous family of functions at } u \text {. }
\end{aligned}
$$

Then the function $F$ is locally lipschitzian on $\Omega$. Moreover, there is a dense $G_{\delta}$ subset $T \subset V$, at every point $u$ of which

$$
\text { the filter } G_{u} \text { converges in } V^{*} \text {-norm, }
$$

$$
F \text { is Fréchet-differentiable, }
$$

the derivative $F^{\prime}(u)$ being exactly the limit of $G_{u}$, and being a continuous function of $u$ on $T$.

Proof. Assumptions (2.49) and (2.50) imply (2.7), (2.8) and (2.26) at every point of $\Omega$. By Proposition 2.4 the function $F$ is locally lipschitzian on $\Omega$. By Theorem 1.2, for every $\epsilon>0$, the set $S_{\epsilon}$ of points where $F$ is locally $\epsilon$ supported is dense in $\Omega$. By Proposition $2.2, S_{\epsilon}$ is contained in the set $T_{\epsilon}$ of points $u$ where the filter $G_{u}$ has an element of diameter less than $8 \epsilon$. Thus the set $T_{\epsilon}$ is dense in $\Omega$ for every $\epsilon>0$, and by Proposition 2.3 it is open.

We now apply the Baire category theorem to $\Omega$, which is an open subset of a complete metric space $V$, and to the sequence $T_{1 / n}$ of open dense subsets. The intersection

$$
T=\bigcap_{n=1}^{\infty} T_{1 / n}
$$


is a dense $G_{\delta}$ subset of $\Omega$. Let $u \in T$. For every $n \in N$, the filter $G_{u}$ has an element of diameter less than $1 / n$. But this simply means that $G_{u}$ is a Cauchy filter in $V^{*}$. Since $V^{*}$ is norm-complete, the filter $G_{u}$ converges towards $u^{*} \in V^{*}$. By Proposition 2.4, the function $F$ admits $u^{*}$ as Fréchet-derivative at $u$.

There only remains to prove the continuity. Let $u \in T$ and $\epsilon>0$ be given. As $u \in T_{\epsilon}$, there exists in $G_{u}$ an element $A_{u}^{*}(\eta, \theta)$ with diameter less than $\epsilon$. By formula (2.36) we can find $\delta>0$ such that, for $\|v-u\| \leqslant \delta$, there is an element $A_{v}^{*}(\delta, \beta)$ of filter $G_{v}$ contained in $A_{u}^{*}(\eta, \theta)$. But $F^{\prime}(u) \in \overline{A_{u}^{*}(\eta, \theta)}$, and if $v \in T$, then $F^{\prime}(v) \in \overline{A_{v}^{*}(\delta, \beta)}$. Hence

$$
\|u-v\| \leqslant \delta \text { and } v \in T \Rightarrow F^{\prime}(v) \in \overline{A_{u}^{*}(\eta, \theta)}
$$

This yields the desired result

$$
\|u-v\| \leqslant \delta \text { and } v \in T \Rightarrow\left\|F^{\prime}(v)-F^{\prime}(u)\right\|_{*} \leqslant \epsilon .
$$

Let us give an application.

THEOREM 2.6. Let $V$ be a Banach space satisfying condition $(\mathrm{H})$, and $G$ a lower semicontinuous convex function on $V$. The interior of $\operatorname{dom} G$ then contains a dense $G_{\delta}$ subset $T$ at every point of which $G$ is Fréchet-differentiable, the mapping $G^{\prime}: T \rightarrow V^{*}$ being continuous.

Proof. We shall use some tools of convex analysis (see [12] for instance). Denote by $G^{*}$ the conjugate convex function of $G$ on $V$ :

$$
G^{*}\left(u^{*}\right)=\sup _{u \in V}\left\langle u, u^{*}\right\rangle-G(u)
$$

We know that $G=G^{* *}$, that is

$$
G(u)=\sup _{u^{*} \in V^{*}}\left\langle u, u^{*}\right\rangle-G^{*}\left(u^{*}\right)
$$

The result now follows from Theorem 2.5, with $X=V^{*}$ and $F=-G$, provided we check assumptions (2.49) and (2.50) for the function

$$
f\left(u, u^{*}\right)=G^{*}\left(u^{*}\right)-\left\langle u, u^{*}\right\rangle
$$

with $\Omega=\operatorname{do} G$. Let $u \in \operatorname{do̊m} G$.

Of course, $f_{v}^{\prime}\left(v, v^{*}\right)$ is just the linear functional $v^{*}$ on $V$, so that (2.49) and (2.50) will both be implied by the following property: for every $b \in \mathbf{R}$, there exist $\eta>0$ and $C>0$ such that

$$
\|u-v\| \leqslant \eta / 2, \quad f\left(v, v^{*}\right) \leqslant b \Rightarrow\left\|v^{*}\right\|_{*} \leqslant C .
$$

So let us prove (2.59). It follows from the assumption on $G$ that it is continuous on the interior of its effective domain (e.g. [12, Corollary 1.2.5]), 
and hence bounded on a neighbourhood of $u$. There exist $a \in R$ and $\eta>0$ such that

$$
\|v-u\| \leqslant \eta \Rightarrow G(v) \leqslant a
$$

Taking conjugates

$$
G^{*}\left(v^{*}\right) \geqslant \eta\left\|v^{*}\right\|_{*}+\left\langle u, v^{*}\right\rangle-a, \quad \forall v \in V .
$$

Now write the assumption $f\left(v, v^{*}\right) \leqslant b$

$$
b \geqslant G^{*}\left(v^{*}\right)-\left\langle v, v^{*}\right\rangle .
$$

Adding (2.61) and (2.62)

$$
a+b \geqslant \eta\left\|v^{*}\right\|_{*}+\left\langle u-v, v^{*}\right\rangle .
$$

If we now assume that $\|u-v\| \leqslant \eta / 2$, this yields

$$
a+b \geqslant \eta\left\|v^{*}\right\|_{*}-1 / 2 \eta\left\|v^{*}\right\|_{*}
$$

hence (2.59) with $C=2(a+b) / \eta$.

In the terminology of Asplund [1], every Banach space satisfying assumption $(\mathrm{H})$ is a strong differentiability space. This does not follow from the known differentiability theorems, inasmuch as $V$ is not assumed to be weakly compactly generated, and in fact answers an open question [7, VII, 4A, (7)].

3. Application to optimization. The aim of this section is to investigate existence and stability properties for solutions of optimization problems depending on parameters. To that purpose, we shall use the results and notations of the preceding section. Let us review (2.1)-(2.4) from the point of view of optimization theory.

We are interested in the optimization problem:

$$
\inf _{x \in X} f(0, x)
$$

which by (2.3) is nontrivial. We look for optimal solutions, i.e. points $\bar{x}$ where the minimum is attained:

$$
f(0, \bar{x}) \leqslant f(0, x) \quad \forall x \in X
$$

We want to know whether an optimal solution exists at all, and if so, whether it is unique. We also want to know something about the behaviour of the minimizing filter $F_{0}$ defined by (2.4). Let us call the problem well-behaved when there exists a unique optimal solution $\bar{x}$, and the minimizing filter converges to $\bar{x}$ for some topology on $X$ : this ensures easy practical computation of the theoretical solution. This is not always the case, except for stringent assumptions 
which we do not make ( $X$ should be a compact convex set and $f(0, \cdot)$ should be lower semicontinuous and strictly convex). But we shall prove that, for certain types of perturbed problems, it is almost always the case.

If $f(\cdot, x)$ is continuous in $u$ for every fixed $x$, the optimization problem:

$$
\inf _{x \in X} f(u, x)
$$

appears as a perturbed problem associated with $\left(P_{0}\right) ; F(u)$ defined by $(2.2)$ will be its value. The nearer $u$ to the origin, the smaller the perturbation. This is of particular interest in the case where the perturbed problems are better-behaved than the original one, because we may simply choose $u$ small enough for the perturbation to be insignificant for all practical purposes, and deal with $\left(P_{u}\right)$ instead of $\left(P_{0}\right)$.

The following definition will be the main tool in adjusting Theorem 2.5 to this new framework:

Definition 3.1. Let $\phi: X \rightarrow Y$ be a continuous mapping between topological spaces. It will be called proper iff every filter $\Phi$ on $X$ whose image by $\phi$ converges admits a cluster point.

We refer to Bourbaki [6] for further properties of proper maps, among which we cite the following (recall that a mapping is closed iff it sends every closed set onto a closed set):

Proposition 3.2. Let $X$ be Hausdorff. Then a continuous map $\phi$ is proper iff it is closed and $\phi^{-1}(\{y\})$ is compact for every $y \in Y$.

Proposition 3.3. Let $\phi$ be continuous and injective. The following are equivalent:

(3.2) $\phi$ is proper,

(3.3) $\phi$ is closed,

(3.4) $\phi$ is a homeomorphism of $X$ onto a closed subset of $Y$.

We now state a general theorem on perturbations. It is an easy consequence of Theorem 2.5, but we shall show that it yields all the examples known up to date, and more.

THEOREM 3.4. Let $V$ be a Banach space satisfying condition $(\mathrm{H}), X$ a topological space, and $f$ a function on $V \times X$ such that

$$
\forall u \in V, \quad F(u)=\inf _{x \in X} f(u, x)>-\infty .
$$

Assume there is an open subset $\Omega \subset V$, every point $u$ of which has the following properties:

(3.6) there is a $B_{\eta, \theta}^{*} \in H_{u}$ which is norm-bounded in $V^{*}$, 
(3.7) there is an $A_{\theta} \in F_{u}$ such that $\left\{f_{v}^{\prime}(\cdot, x) \mid x \in A_{\theta}\right\}$ is an equicontinuous family of functions at $u$,

(3.8) the function $x \mapsto f(u, x)$ is lower semicontinuous on $X$,

(3.9) the mapping $x \mapsto f_{v}^{\prime}(u, x)$ from $X$ to $V^{*}$ is continuous and proper.

Then $F$ is locally lipschitzian on $\Omega$. Moreover, there exists a dense $G_{\delta}$ set $T \subset \Omega$, at every point $u$ of which:

(3.10) $F$ is Fréchet-differentiable and $G_{u}$ converges to $F^{\prime}(u)$ in $V^{*}$,

(3.11) the set $S_{u}$ of optimal solutions for $\left(P_{u}\right)$ is nonempty, and is exactly the set of cluster points of the minimizing filter $F_{u}$,

(3.12) every $x \in S_{u}$ satisfies the necessary condition for optimality, $f_{v}^{\prime}(u, x)=F^{\prime}(u)$.

Proof. Formula (3.6) has been copied from (2.49) and formula (3.7) from (2.50), so that we may apply Theorem 2.5. Hence formula (3.10).

Denote by $\phi$ the mapping $x \mapsto f_{v}^{\prime}(u, x)$. The image of $F_{u}$ by $\phi$ is finer than $G_{u}$, and as $G_{u}$ converges to $F^{\prime}(u)$, so does $\phi\left(F_{u}\right)$. As $\phi$ is proper it follows that the set $S_{u}$ of cluster points of $F_{u}$ is nonempty. As $\phi$ is continuous, it sends $S_{u}$ on the limit of $G_{u}$, hence

$$
\forall x \in S_{u}, \quad f_{v}^{\prime}(u, x)=F^{\prime}(u) .
$$

It only remains to prove that $S_{u}$ is the set of optimal solutions for $\left(P_{u}\right)$. Let $\bar{x}$ be an optimal solution for $\left(P_{u}\right)$; then $f(u, \bar{x})=F(u)$, hence $\bar{x} \in A_{\theta}$ for every $\theta>0$; this proves that $\bar{x}$ belongs to the intersection of all elements of $F_{u}$, hence $\bar{x} \in S_{u}$. Conversely, let $\bar{x}$ be a cluster point of $F_{u}$. By definition, there exists a filter $F_{u}^{\prime}$ which is finer than $F_{u}$ and converges to $\bar{x}$. By assumption (3.8), we have

$$
f(u, \bar{x}) \leqslant \underline{\lim } f(u, x) \text { as } x \stackrel{F_{u}^{\prime}}{\longrightarrow} \bar{x} .
$$

But the filter $F_{u}$ is just the inverse image by $f\left(u,{ }^{\circ}\right)$ of the filter of neighbourhoods of $F(u)$ in $\mathbf{R}$. The image by the same mapping of any finer filter, such as $F_{u}^{\prime}$, must perforce converge to $F(u)$ :

$$
F(u)=\lim f(u, x) \quad \text { as } x \stackrel{F_{u}^{\prime}}{\longrightarrow} \bar{x} .
$$

Comparing (3.14) and (3.15), we get

$$
f(u, \bar{x}) \leqslant F(u)
$$

As the converse inequality is true by definition (formula (2.2)) equality holds in (3.16), which shows that $\bar{x}$ is an optimal solution of $\left(P_{u}\right)$ and concludes the proof. 
COROLlaRY 3.5. The set-valued mapping $u \rightarrow S_{u}$ from $T$ to $X$ is upper semicontinuous with compact nonempty values.

Proof. By its definition, $S_{u}$ is clearly closed, either as the set of points where a lower semicontinuous function attains its minimum, or as the set of cluster points of a filter (see (3.11)). Moreover, Theorem 3.4 states that

$$
\varnothing \neq S_{u} \subset\left\{x \mid f_{v}^{\prime}(u, x)=F^{\prime}(u)\right\} \quad \forall u \in T .
$$

As the mapping $f_{v}^{\prime}(u, \cdot)$ is proper, the right-hand set is compact (Proposition 3.2), and so are all its closed subsets, particularly $S_{u}$.

To prove upper semicontinuity on $T$, take any closed subset $A$ of $X$ not intersecting $S_{u}$. We have to prove that $A \cap S_{v}=\varnothing$ for all $v \in T$ in a neighbourhood of $u$. Assume it is not so. Then, for every $\theta>0$, the set

$$
S_{\theta}=\bigcup S_{v} \text { for } v \in T \text { and }\|v-u\| \leqslant \theta
$$

intersects $A$. Thus the family $S_{\theta}, \theta>0$, generates a filter on $X$ which we denote by $\Theta_{u}$.

Recall from the preceding section $((2.29) \Rightarrow(2.35)$ with $w=u)$ that for any $\epsilon>0$, there exists $\eta>0$ such that

$$
\|u-v\| \leqslant \eta \text { and } f(v, x) \leqslant F(v)+\frac{\epsilon}{3} \Rightarrow f(u, x) \leqslant F(u)+\epsilon .
$$

This implies that $S_{\eta}$ is a member of $F_{u}$, and hence that $\Theta_{u}$ is finer than $F_{u}$. Therefore, the image of $\Theta_{u}$ by the proper mapping $f_{v}^{\prime}(u, \cdot)$ is finer than $G_{u}$, which converges to $F^{\prime}(u)$ since $u \in T$. Hence $\Theta_{u}$ has one cluster point $\bar{x}$ at least; as $A$ is closed, $\bar{x} \in A$; since $\Theta_{u}$ is finer than $F_{u}, x$ is a cluster point of $F_{u}, \bar{x} \in$ $S_{u}$. Hence $\bar{x} \in A \cap S_{u}$, which was assumed to be empty.

COROllary 3.6. Assume in addition that, for every $u \in \Omega$, the mapping $f_{v}^{\prime}(u, \cdot)$ is injective. Then, for every $u \in T$, the problem $\left(P_{u}\right)$ has a unique optimal solution $s(u)$, and the minimizing filter $F_{u}$ converges towards $s(u)$. Moreover, the mapping s: $T \rightarrow X$ is continuous.

Proof. Indeed, all optimal solutions of $\left(P_{u}\right)$ have to satisfy equation (3.12), which has at most one solution. So there cannot be more than one optimal solution, and we know there is at least one. The set $S_{u}$ is then reduced to the singleton $\{s(u)\}$. As the set-valued mapping $u \rightarrow\{s(u)\}$ is upper semicontinuous, the map $u \rightarrow s(u)$ is continuous.

Thus $s(u)$ is the only cluster point of $F_{u}$. We conclude that $F_{u}$ converges to $s(u)$. Indeed, if it were not so, there would be a neighbourhood $V$ of $s(u)$ in $X$ such that every element of $F_{u}$ intersects $C V$. These intersections generate a filter $F_{u}^{\prime}$ which is finer than $F_{u}$. Its image by the proper mapping $f_{v}^{\prime}(u, \cdot)$ is 
finer than $G_{u}$, which converges to $F^{\prime}(u)$. Hence $F_{u}^{\prime}$ has a cluster point $\bar{x}$. But $\bar{x}$ has to be a cluster point for the coarser filter $F_{u}$, and it cannot belong to the interior of $V$. Hence $x \neq s(u)$, and we have two cluster points for $F_{u}$, a contradiction.

EXAMPLE 1. Linear perturbations. Let $X$ be a topological space and $V$ a Banach space which satisfies condition $(\mathrm{H})$. Let $g: X \rightarrow \mathbf{R}$ be lower semicontinuous and bounded from below, and let $A: X \rightarrow V^{*}$ be continuous and proper. Assume $A(X)$ is norm-bounded in $V^{*}$. Define $f: V \times X \rightarrow \mathbf{R}$ by

$$
f(u, x)=g(x)+\langle A x, u\rangle .
$$

Since $f_{v}^{\prime}(u, x)$ is just $A x$, all the assumptions of Theorem 3.4 are trivially satisfied with $\Omega=V$. We conclude that

Proposition 3.7. Consider the optimization problem

$$
\inf _{x \in X} g(x)+\langle A x, u\rangle
$$

with value $F(u)$. Then $F$ is locally lipschitzian on $V$ and there exists a dense $G_{\delta}$ subset $T \subset V$, at every point $u$ of which:

(3.21) $F$ is Fréchet-differentiable,

(3.22) the set $S_{u}$ of optimal solutions for $\left(P_{u}\right)$ is compact nonempty,

(3.23) $\forall x \in S_{u}, A x=F^{\prime}(u)$,

(3.24) the restriction to $T$ of the mapping $F^{\prime}: V \rightarrow V^{*}$ is continuous,

(3.25) the set-valued mapping $u \mapsto S_{u}$ from $T$ to $X$ is u.s.c.

For instance, if $X$ is a closed bounded subset of some Banach space $Y$, we can take for $V$ the Banach space $Y^{*}$, inasmuch as it satisfies condition $(\mathrm{H})$, and for $A$ the canonical isometry from $X$ into $V^{* *}$, which is obviously continuous and proper. It is even linear and injective, so that we can apply Corollary 3.6 and get

Corollary 3.8. Let $X$ be a closed bounded subset of a Banach space $Y$ and $g$ a lower semicontinuous function on $X$, bounded from below. Assume that the dual $Y^{*}$ of $Y$ satisfies condition $(\mathrm{H})$. With every $y^{*} \in Y^{*}$ associate the optimization problem:

$$
\inf _{x \in X} g(x)+\left\langle x, y^{*}\right\rangle
$$

with value $F\left(y^{*}\right)$. Then $F$ is a concave continuous function on $Y^{*}$, and there exists a dense $G_{\delta}$ subset $T \subset Y^{*}$, on which the Fréchet-derivative $F^{\prime}$ is well defined and continuous. Moreover, for every $y^{*} \subset T$, there is a single optimal 
solution $s\left(y^{*}\right)$ for $\left(P_{y^{*}}\right)$, depending continuously on $y^{*}$. It is the limit of all minimizing sequences

$$
g(x)+\left\langle x_{n}, y^{*}\right\rangle \rightarrow F\left(y^{*}\right) \Rightarrow\left\|s\left(y^{*}\right)-x_{n}\right\| \rightarrow 0
$$

and satisfies the necessary condition for optimality

$$
s\left(y^{*}\right)=F^{\prime}\left(y^{*}\right) \text {. }
$$

Recall that $Y$ is reflexive if and only if $Y^{*}$ can be equivalently renormed in a Fréchet-differentiable way [8, II.3, V.5]. This is an important case where the assumption of Corollary 3.8 is satisfied.

Applying Proposition 3.7 to the special case where $X$ is a closed bounded subset of $V^{*}$ and $A$ is the identity map, one gets an earlier result of Asplund [1].

By a classical argument (Asplund [1]), we get another consequence of Proposition 3.7.

COROLlaRY 3.8 BIS. Let $Y$ be a Banach space whose dual $Y^{*}$ satisfies condition $(\mathrm{H})$. Then every closed convex and bounded subset of $Y$ is the closed convex hull of its strongly exposed points.

Proof. We apply Corollary 3.8 to the special case where $X$ is convex and $g$ is zero. The conclusion then reads that there is a dense $G_{\delta}$ subset $T \subset Y^{*}$, every point $y^{*}$ of which strongly exposes some point $s\left(y^{*}\right) \in X$.

Let $S$ be the set of strongly exposed points of $X$, and assume $X \neq \overline{c o} S$. Then there is some point $\bar{x} \in X$ which does not belong to $\overline{c o} S$. Applying the Hahn-Banach theorem, there is some $\bar{y}^{*} \in Y^{*}$ such that

$$
\sup _{s \in S}\left\langle\bar{y}^{*}, s\right\rangle=\alpha\left\langle\left\langle\bar{y}^{*}, \bar{x}\right\rangle\right.
$$

Let $\epsilon=\left\langle\bar{y}^{*}, x\right\rangle-\alpha$, and $M=\sup _{x \in X}\|x\|$. If $y^{*} \in Y^{*}$ is such that $\left\|y^{*}-\bar{y}^{*}\right\|_{*}<\epsilon / 2 M$, we get

$$
\sup _{s \in S}\left\langle y^{*}, s\right\rangle\left\langle\alpha+\epsilon / 2 \leqslant\left\langle\bar{y}^{*}, \bar{x}\right\rangle-\epsilon / 2<\left\langle y^{*}, \bar{x}\right\rangle .\right.
$$

This means that the open ball of radius $\epsilon / 2 M$ around $\bar{y}^{*}$ contains no continuous linear functional which attains its maximum on $X$ at some strongly exposed point. But this contradicts the fact that the set $T$ of such functionals is dense in $Y^{*}$. $\quad$ o

It is a theorem of Lindenstrauss (Diestel $[8, \mathrm{~V} .6]$ ) that every convex and weakly compact subset of a Banach space is the closed convex hull of its strongly exposed points. This theorem and Corollary 3.8 bis have different scopes, except in the case of reflexive Banach spaces, where both apply to yield the same result. 
EXAMPLE 2. Perturbations by norm functions. Let again $V$ be a Banach space and $V^{*}$ its dual. In the sequel, we shall always assume that both $V$ and $V^{*}$ are uniformly convex. This means that for every $\epsilon>0$, there exist $\eta>0$ and $\eta^{*}>0$ such that

$$
\|u\| \leqslant 1, \quad\|v\| \leqslant 1, \quad\|u-v\| \geqslant \epsilon \Rightarrow\|u+v\| \leqslant 2(1-\eta),
$$

$\left\|u^{*}\right\|_{*} \leqslant 1, \quad\left\|v^{*}\right\|_{*} \leqslant 1, \quad\left\|u^{*}-v^{*}\right\|_{*} \geqslant \epsilon \Rightarrow\left\|u^{*}+v^{*}\right\|_{*} \leqslant 2\left(1-\eta^{*}\right)$.

Such is the case, for instance, for Hilbert spaces, and for $L^{p}$ spaces with $1<p<\infty$.

It is known that all uniformly convex Banach spaces are reflexive. Moreover, we shall use the following two lemmas:

LEMmA 3.9. The norm of $V$ is Fréchet-differentiable at every point $u \neq 0$, and the restriction of its derivative $j$ to any closed bounded subset of $V$ not containing the origin is uniformly continuous.

Proof. We shall use the language and some of the results of convex analysis. The mapping $u \mapsto\|u\|$ is continuous and convex, and we denote by $J(u)$ its subgradient at $u \neq 0$ :

$$
J(u)=\left\{u^{*} \in V^{*}\left\|u^{*}\right\|_{*}=1,\left\langle u, u^{*}\right\rangle=\|u\|\right\} .
$$

Let $B$ be a closed bounded subset with $0 \notin B$. We shall prove that, for every $\epsilon>0$, there exists $\delta>0$ such that $(3.31)(u, v) \in B \times B, \quad\left(u^{*}, v^{*}\right) \in J(u) \times J(v), \quad\|u-v\| \leqslant \delta \Rightarrow\left\|u^{*}-v^{*}\right\| \leqslant \epsilon$.

It follows immediately that $J$ is in fact a singleton, $J(u)=\{j(u)\}$, the mapping $j: V \rightarrow V^{*}$ being uniformly continuous on $B$. By [12, Proposition I.5.3] the norm is Gâteaux-differentiable at $u$ with derivative $j(u)$, and by $[11$, Definition 2.1] it is even Fréchet-differentiable.

It only remains to prove (3.31). Suppose it were not true. Then there would exist sequences $u_{n}, v_{n}$ in $B, u_{n}^{*}, v_{n}^{*}$ in $V^{*}$, such that

$$
u_{n}^{*} \in J\left(u_{n}\right), \quad v_{n}^{*} \in J\left(v_{n}\right), \quad\left\|u_{n}-v_{n}\right\| \rightarrow 0,\left\|u_{n}^{*}-v_{n}^{*}\right\|_{*}>\epsilon .
$$

Applying (3.29), we get

$$
\left\langle u_{n}^{*}+v_{n}^{*}, u_{n}\right\rangle \leqslant\left\|u_{n}\right\| \cdot\left\|u_{n}^{*}+v_{n}^{*}\right\|_{*} \leqslant 2\left(1-\eta^{*}\right)\left\|u_{n}\right\| .
$$

Using the definition (3.30) of $J$, this becomes

$$
\left\|u_{n}\right\|+\left\|v_{n}\right\|+\left\langle v_{n}^{*}, u_{n}-v_{n}\right\rangle \leqslant 2\left(1-\eta^{*}\right)\left\|u_{n}\right\| .
$$

Transforming the left-hand side by the triangle inequality 


$$
2\left\|u_{n}\right\|-\left\|v_{n}-u_{n}\right\| \leqslant 2\left(1-\eta^{*}\right)\left\|u_{n}\right\|+\left\langle v_{n}-u_{n}, v_{n}^{*}\right\rangle
$$

$$
\leqslant 2\left(1-\eta^{*}\right)\left\|u_{n}\right\|+\left\|v_{n}-u_{n}\right\| .
$$

As $n$ goes to infinity, $\left\|u_{n}-v_{n}\right\| \rightarrow 0$; since $u_{n} \in B$, its norm is bounded away from zero and from infinity, and the $\left\|u_{n}\right\|$ must have a cluster point $\lambda \in$ ] $0,+\infty[$. Passing to the limit in (3.35), we get a contradiction:

$$
2 \lambda \leqslant 2\left(1-\eta^{*}\right) \lambda . \quad \square
$$

The mapping $j: V \backslash\{0\} \rightarrow V^{*}$ is usually called the duality map from $V$ to $V^{*}$. We have seen (3.30) that it is characterized by the equalities

$$
\forall u \neq 0,\|j(u)\|_{*}=1 \text { and }\langle j(u), u\rangle=\|u\| .
$$

Of course the same lemma applies to $V^{*}$, with a duality map $j^{*}$ from $V^{*}$ to $V$. Now for the next lemma.

LEMMA 3.10. The restriction of the duality mapping $j$ to any closed bounded subset of $V$ not containing the origin is a proper map.

Proof. Denote by $B$ a closed bounded subset of $V$ such that $B \nexists 0$. Let $F$ be a filter on $B$ such that $j(F)$ converges to some point $u^{*}$ in $V^{*}$. As $B$ is bounded and $V$ reflexive, there is a finer filter $G$ on $B$ such that $v$ G-converges to some point $u$ in the weak topology of $V$, and $\|v\| G$-converges to some number $\rho$ in $R$. As the norm is weakly lower semicontinuous, we have

$$
\|u\| \leqslant \lim _{G}\|u\| \text {. }
$$

Taking (3.37) into account, we have

$$
\lim _{G}\|v\|=\lim _{G}\langle j(v), v\rangle=\left\langle u^{*}, u\right\rangle
$$

and hence

$$
\|u\| \leqslant \lim _{G}\|v\|=\left\langle u^{*}, u\right\rangle .
$$

But we know that $j(F)$ converges to $u^{*}$ and that $\|j(v)\|_{*}=1$ for every $v \neq 0$. We conclude that $\left\|u^{*}\right\|_{*}=1$, and finally

$$
\lim _{G}\|u\|=\|u\| \text {. }
$$

We have proved that $v \stackrel{G}{\rightarrow} u$ weakly and $\|v\| \stackrel{G}{\rightarrow}\|u\|$. It is a wellknown fact from the theory of uniformly convex Banach spaces that this implies that $v \stackrel{G}{\rightarrow} u$ strongly. Indeed, if it were not so, the filter $G$ would not be Cauchy, and hence there would exist an $\epsilon>0$ such that all elements of $G$ had diameter greater than $2 \epsilon\|u\|$. 


$$
\forall A \in G, \quad \exists v \in A, \quad \exists w \in A:\|v-w\| \geqslant 2 \epsilon\|u\| .
$$

By the triangle inequality, noting that $A \subset B \nsupseteq 0$ :

$$
\|v /\| v\|-w /\| w\|\| \geqslant\|v-w\| /\|v\|-\|w\|(1 /\|v\|-1 /\|w\|) \text {. }
$$

As $\|v\| \stackrel{G}{\rightarrow}\|u\|$ and $\|w\| \stackrel{G}{\rightarrow}\|u\|$, the last term goes to zero. Hence there is a $D \in G$ such that, for every $A \in G$, there exist $v$ and $w$ in $A \cap D$ with

$$
\|v /\| v\|-w /\|_{w}\|\| \geqslant \epsilon .
$$

Using (3.28), this implies

$$
\|v /\| v\|+w /\| w\|\| \leqslant 2(1-\eta)
$$

Taking scalar products with $j(u)$

$$
\langle v /\|v\|+w /\|w\|, j(u)\rangle \leqslant 2(1-\eta) .
$$

Since we know that $G$ converges weakly to $u$, we can take the G-limit of the left-hand side to get the desired contradiction

$$
2\langle u /\|u\|, j(u)\rangle=2 \leqslant 2(1-\eta) .
$$

We have thus proved that $G$ converges in $B$, and hence that the coarser filter $F$ has a cluster point.

With these preliminary lemmas on uniformly convex Banach spaces out of the way, we now proceed to the statement and proof of our main example.

THEOREM 3.11. Let $V$ be a Banach space and $V^{*}$ its dual, both uniformly convex, and $X$ a topological space. Let $A$ be a continuous proper mapping from $X$ into $V$, with $A(X)$ bounded. We consider the family of optimization problems:

$$
\inf _{x \in X} g(x)+h(\|u-A x\|)
$$

where $g$ is a lower semicontinuous function on $X$, bounded from below, and $h$ a continuous function on $[0,+\infty]$, with derivative $h^{\prime}$ continuous and nonzero on $] 0,+\infty\left[\right.$. We denote by $F(u)$ the value of $\left(P_{u}\right)$ and assume that $h(0)=0$.

Then $V$ can be partitioned into a closed set $G$ and an open set $\Omega$. At every point $u$ of $G$, there is an optimal solution $x$ for $\left(P_{u}\right)$ satisfying $A x=u$. There is a dense $G_{\delta}$ subset $T$ of $\Omega$, at every point $u$ of which $\left(P_{u}\right)$ has a compact nonempty set of optimal solutions, all of which satisfy $h^{\prime}(\|u-A x\|) j(u-A x)=$ $F^{\prime}(u)$.

Proof. We define $G$ as the set of all points $u \in V$ such that $\left(P_{u}\right)$ has an optimal solution satisfying $A x=u$. Clearly, it is the image by $A$ of the set 


$$
\{x \in X \mid g(x) \leqslant F(A x)\}
$$

This is a closed set, because $g$ is lower semicontinuous and $F$, being the pointwise infimum of a family of continuous functions, is upper semicontinuous. Since $A$ is proper, it maps a closed set onto a closed set (Proposition 3.2). Hence $G$ is closed.

It is now straightforward to apply Theorem 3.4 to $\Omega=C G$ and $f(u, x)=$ $g(x)+h(\|u-A x\|)$. If $u$ belongs to $\Omega$, which is open in $V$, we can find an element $D$ of the minimizing filter $F_{u}$ and a ball $V$ of radius $\epsilon>0$ around $u$ such that

$$
\forall x \in D, \quad \forall v \in V, \quad A x \neq v .
$$

Indeed, if it were not so, the image by $A$ of any element of $F_{u}$ would intersect any neighbourhood of $u$. But this means that there exists some filter which is finer than $F_{u}$ and whose image by $A$ converges towards $u$. Since $A$ is proper, this filter would then have some cluster point $\bar{x}$ satisfying $A \bar{x}=u$. But $\bar{x}$ would also be a cluster point for the coarser filter $F_{u}$, thus contradicting the fact that $u \notin G$.

From (3.48) we conclude that for every $x \in D$, the function $f(;, x)$ is Fréchet-differentiable on the neighbourhood $V$, with derivative $f_{v}^{\prime}(v, x)=$ $h^{\prime}(\|v-A x\|) j(v-A x)$. Since $h^{\prime}$ is continuous on $] 0,+\infty[$ it is bounded and uniformly continuous on every compact subset. But we know that $A(X)$ is bounded, and it follows from (3.48) that $\|v-A x\| \geqslant \epsilon / 2$ for every $x$ in $D$ and $v$ in $V / 2$. This proves condition (3.6), and, together with Lemma 3.9, condition (3.7). Condition (3.8) is clearly satisfied, and we are left with condition (3.10), i.e. to prove that the map $x \longmapsto f_{v}^{\prime}(u, x)=h^{\prime}(\|u-A x\|) j(u-A x)$ is proper.

Let $E$ be any filter on $X$ such that its image by $f_{v}^{\prime}\left(u,{ }^{\circ}\right)$ converges in $V$. Its image by the mapping $x \mapsto h^{\prime}(\|u-A x\|)$ is bounded in $R$, because $A(X)$ is bounded, and so must have cluster points; by taking a finer filter if necessary, we may even assume that it converges. Either its limit is zero or not. The first case, $h^{\prime}(\|u-A x\|) \rightarrow 0$, can only occur if $A x \rightarrow u$, because $h^{\prime}(t) \neq 0$ for every $t>0$; since $A$ is proper, it implies that $E$ has a cluster point. The only case left to consider is when $\lim h^{\prime}(\|u-A x\|) \neq 0$ and $\lim A x-u \neq 0$. Then, on some closed subset $X^{\prime}$ of $X$ not containing the origin, $E$ induces a filter the image of which by the mapping $x \mapsto j(u-A x)$ converges; by Lemma 3.10 we conclude that this filter must have a cluster point. Thus $E$ always has a cluster point, and we are through with the proof.

To keep down the length of the theorem, we have spared the reader the detailed properties of $F$. It is locally lipschitzian on $\Omega$, by Theorem 3.4. For every $u$ in $G$, we have $F(u)=g(u)$. It follows that the restriction of $F$ to $G$ is 
continuous (1.s.c. because it coincides with $g$, and u.s.c. because it is a pointwise infimum of continuous functions). Recall also that $u \mapsto S_{u}$ is upper semicontinuous as a set-valued mapping from $T$ to $X$.

The general result can be refined in two ways, either seeking uniqueness of the optimal solution on $T$, or getting rid of the exceptional set $G$. For uniqueness, we use Corollary 3.6.

Corollary 3.12. Assumptions of Theorem 3.11. Assume in addition that $A$ is injective, and one of the following:

$$
g=0,
$$

or

$$
\left.h^{\prime} \text { is injective on }\right] 0,+\infty[\text {. }
$$

Then, for every $u$ in $T$, there is a unique solution $s(u)$ for $\left(P_{u}\right)$. The mapping s: $T \rightarrow X$ is continuous, and all minimizing sequences for $\left(P_{u}\right)$ converge to $s(u)$.

Proof. Suppose there were two optimal solutions $x$ and $y$ for $\left(P_{u}\right), u \in$ T. Then we would have

$$
\begin{aligned}
g(x)+h(\|u-A x\|) & =g(y)+h(\|y-A x\|) ; \\
h^{\prime}(\|u-A x\|) j(u-A x) & =h^{\prime}(\|u-A y\|) j(u-A y) .
\end{aligned}
$$

If (3.49) is fulfilled, it follows from (3.51) and the fact that $h$ is strictly monotone that $\|u-A x\|=\|u-A y\|$. If (3.50) is fulfilled, it follows from (3.52) by taking norms that $\|u-A x\|=\|u-A y\|$. In any case we have

$$
\|u-A x\|=\|u-A y\| \text { and } j(u-A x)=j(u-A y) .
$$

This of course means that $u-A x=u-A y$, and hence $x=y$.

The exceptional set $G$ cannot be dispensed with if the function $v \mapsto h(\|v\|)$ is not differentiable at the origin. It can even be the whole space $V$, as in the case where $X=V, A$ is the identity on $V, h$ is the identity on [0, $+\infty]$ and $g$ satisfies the Lipschitz condition $|g(u)-g(v)| \leqslant\|u-v\|$. Problem $\left(P_{u}\right)$ then always has $x=u$ as an optimal solution, and $F(u)=g(u)$; there is no differentiability property of $F$ nor any nice first-order condition for optimality. It is, of course, more interesting to know when we can take $G=\varnothing$ :

Corollary 3.13. Assumptions of Theorem 3.11. Assume in addition that

$$
h(t) / t \rightarrow 0 \text { when } t \rightarrow 0 \text {. }
$$

Then the conclusions hold with $G=\varnothing$ and $\Omega=V$. 
Of course Corollaries 3.12 and 3.13 can be combined. For instance, take for $X$ a bounded closed subset of $V$, for $A: X \rightarrow V$ the canonical injection, and for $h$ the function $t \rightarrow t^{p}$ (or $t \rightarrow-t^{p}$ ), with $1<p<\infty$. Then both corollaries hold, and we get the following statements; we always denote by $V$ a Banach space with dual $V^{*}$, both uniformly convex, by $X$ a bounded closed subset of $V$, by $g$ a lower semicontinuous function on $X$, bounded from below:

Proposition 3.14. Under the above assumptions, consider the optimization problem:

$$
\inf _{x \in X} g(x)+\|x-u\|^{p}
$$

with value $F(u)$. There is a dense $G_{\delta}$ subset $T$ of $V$, at every point $u$ of which $F$ is Fréchet-differentiable and all minimizing sequences for $\left(P_{u}\right)$ converge to a single optimal solution. This solution depends continuously on $u$ in $T$, and satisfies the first-order condition for optimality:

$$
p\|x-u\|^{p-1} j(x-u)=F^{\prime}(u) .
$$

Taking $g=0$, we get the nearest point problem:

COROLlary 3.15. Denote by $d(u)=\inf _{x \in X}\|u-x\|$ the distance from $u$ to $X$. There is a dense $G_{\delta}$ subset of $V$, at every point of which $d$ is Fréchetdifferentiable and is attained at a single point of $X$.

Proposition 3.16. Under the above assumptions, consider the optimization problem:

$$
\inf _{x \in X} g(x)-\|x-u\|^{p}
$$

with value $F(u)$. There is a dense $G_{\delta}$ subset $T$ of $V$, at every point $u$ of which $F$ is Fréchet-differentiable and all minimizing sequences for $\left(P_{u}\right)$ converge to a single optimal solution. This solution depends continuously on $u$ in $T$, and satisfies the first-order condition for optimality:

$$
p\|x-u\|^{p-1} j(x-u)+F^{\prime}(u)=0 .
$$

Taking $g=0$, we get the farthest point problem:

Corollary 3.17. Denote by $\delta(u)=\sup _{x \in X}\|u-x\|$. There is a dense $G_{\delta}$ subset of $V$, at every point of which $\delta$ is Fréchet-differentiable and is attained at a single point of $X$.

We conclude with some references for this example; the easiest way to classify them is by the assumptions on $h$. Theorem 3.11 assumes mainly that $h$ 
is strictly increasing or strictly decreasing, and is new. The main additional assumption of Corollary 3.12 is that $h$ is strictly convex or strictly concave; of this, only the concave decreasing case was known (Baranger-Temam [4]), the other ones are new. Proposition 3.14 is due to Baranger [3] and Proposition 3.16 to Bidaut [5] . Corollary 3.15 has been proved by Steckin [14], Edelstein [10], and Corollary 3.17 by Edelstein [9], Asplund [1] , [2], all under various, generally weaker, assumptions on the Banach space $V$. A general feature is that there was up to now no common treatment of both types of perturbations, the convex ones and the concave ones.

\section{REFERENCES}

1. E. Asplund, Fréchet-differentiability of convex functions, Acta Math. 121 (1968), 31-47. MR 37 \#6754.

2. - Farthest points in locally uniformly rotund Banach spaces, Israel J. Math. 4 (1966), 213-216. MR 34 \#6480.

3. J. Baranger, Existence de solutions pour des problemes d'optimisation non convexes, J. Math. Pures Appl. 52 (1973), 377-406.

4. J. Baranger and R. Temam, Nonconvex optimization problems depending on a parameter, SIAM J. Control 13 (1974).

5. M. F. Bidaut, Théorèmes d'existence et d'existence en général d'un contrôle optimal pour des systèmes régis par des équations aux dérivées partielles non linéaires, Thèse, Université de Paris, 1973.

6. N. Bourbaki, Eléments de mathématiques. Part. 1. Les structures fondamentales de l'analyse. Livre III. Topologie générale, Chaps. 1, 2, Actualités Sci. Indust., no. 858, Hermann, Paris, 1940. MR 3, 55.

7. M. Day, Normed linear spaces, 3rd rev. ed., Ergebnisse der Math. und ihrer Grenzgebiete, Band 21, Springer-Verlag, Berlin and New York, 1973. MR 49 \#9588.

8. J. Diestel, Geometry of Banach spaces, Lecture Notes in Math., vol. 485, SpringerVerlag, Berlin and New York, 1975.

9. M. Edelstein, Farthest points of sets in uniformly convex Banach spaces, Israel J. Math. 4 (1966), 171-176. MR 34 \#3278.

10. - On nearest points of sets in uniformly convex Banach spaces, J. London Math. Soc. 43 (1968), 375-377. MR 37 \#1954.

11. I. Ekeland, On the variational principle, J. Math. Anal. Appl. 47 (1974), 324353. MR 49 \#11344.

12. I. Ekeland and R. Temam, Convex analysis and variational problems, North-Holland, Amsterdam, 1975.

13. K. John and V. Zizler, Smoothness and its equivalents in weakly compactly generated Banach spaces, J. Functional Analysis 15 (1974), 1-11.

14. S. B. Stečkin, Caractérisation d l'approximation par des sous-ensembles d'espaces vectoriels normes, Rev. Math. Pures Appl. 8 (1963), 5-18.

CENTRE DE RECHERCHE DE MATHÉMATIQUES
SITÉ PARIS-9 DAUPHINE, 75775 PARIS 16, FRANCE 